\title{
Novel Midinfrared Plasmonic Properties of Bilayer Graphene
}

\author{
Tony Low, ${ }^{1, *}$ Francisco Guinea, ${ }^{2}$ Hugen Yan, ${ }^{1}$ Fengnian Xia, ${ }^{1,3}$ and Phaedon Avouris ${ }^{1}$ \\ ${ }^{1}$ IBM T.J. Watson Research Center, 1101 Kitchawan Rd, Yorktown Heights, New York 10598, USA \\ ${ }^{2}$ Instituto de Ciencia de Materiales de Madrid, CSIC, Sor Juana Inés de la Cruz 3, 28049 Madrid, Spain \\ ${ }^{3}$ Yale University, 15 Prospect Street, New Haven, Connecticut 06511, USA
}

(Received 14 September 2013; published 18 March 2014)

\begin{abstract}
We study the midinfrared plasmonic response in Bernal-stacked bilayer graphene. Unlike its monolayer counterpart, bilayer graphene accommodates optically active phonon modes and a resonant interband transition at infrared frequencies. They strongly modify the plasmonic properties of bilayer graphene, leading to Fano-type resonances, giant plasmonic enhancement of infrared phonon absorption, a narrow window of optical transparency, and a new plasmonic mode at higher energy than that of the classical plasmon.
\end{abstract}

DOI: 10.1103/PhysRevLett.112.116801

PACS numbers: 73.20.Mf, 78.67.Wj, 81.05.Xj

Plasmonics [1] is an important subfield of photonics that deals with the excitation, manipulation, and utilization of plasmons-polaritons [2]. It is a key element of nanophotonics [3], metamaterials with novel electromagnetic phenomena [4,5], and also has potential applications in biosensing [6]. Recently, graphene has emerged as a promising platform for plasmonics [7,8]. It has many desirable properties such as gate tunability, extreme light confinement, long plasmon lifetime, and plasmonic resonances in the terahertz to midinfrared (IR) regime [9-14]. Spatially resolved propagating plasmons have been observed with a scanning near-field optical microscope $[15,16]$. Tunable plasmon resonances in the terahertz [17] to IR [18,19] region have been observed in graphene micro- and nanoribbons, and the relative damping pathways have also been studied [19]. Identified applications for graphene plasmonics range from notch filters [18], polarizers, and modulators [17-19] to beam reflect arrays [20], biosensing [21], and IR photodetectors [22] via bolometric effect [23].

In this Letter, we discuss why Bernal $A B$-stacked bilayer graphene is important and interesting in its own right as a plasmonic material. Apart from a few theoretical studies of plasmons in bilayer graphene [24-29], there are still no experimental studies of bilayer graphene plasmonics. The first indication that the plasmonic response in bilayer graphene might be very different than that of the monolayer is its two prominent IR structures in its optical conductivity. IR optical measurements of bilayer graphene reveal a phonon peak at $\hbar \omega \approx 0.2 \mathrm{eV}$, with a strong dependence of peak intensity and Fano-type line shape on the applied gate voltage [30,31]. The interlayer coupling in bilayer graphene also results in two nested bands, which present a set of doping-dependent IR features [32-34]. This interband transition between the two nested bands produced a conductivity peak at $\hbar \omega \approx 0.4 \mathrm{eV}$ in optical IR measurements [35-37]. The impact of these IR structures on the bilayer plasmonic response has not been studied. We found several novel plasmonic effects in bilayer graphene: (i) giant plasmonic enhancement of infrared phonon absorption, (ii) an extremely narrow optical transparency window, and (iii) a new plasmonic mode at higher energy than that of the classical plasmon.

Bilayer graphene arranged in the Bernal $A B$ stacking order is considered, with basis atoms $A_{1}, B_{1}$ and $A_{2}, B_{2}$ in the top and bottom layers, respectively. The intralayer coupling is $\gamma_{0} \approx 3 \mathrm{eV}$, and the interlayer coupling between $A_{2}$ and $B_{1}$ is $\gamma_{1} \approx 0.39 \mathrm{eV}$, an average of values reported in optical IR and photoemission measurements [35-39]. We work within the $4 \times 4$ atomic $p_{z}$ orbitals basis, i.e., $a_{1 \mathbf{k}}^{\dagger}, b_{1 \mathbf{k}}^{\dagger}, a_{2 \mathbf{k}}^{\dagger}, b_{2 \mathbf{k}}^{\dagger}$, where $a_{i}^{\dagger}$ and $b_{i}^{\dagger}$ are creation operators for the $i$ th layer on the $A / B$ sublattices. Within this basis, the Hamiltonian near the $\mathbf{K}$ point can be written as $\quad \mathcal{H}_{k}=v_{f} \pi_{+} I \otimes \sigma_{-}+v_{f} \pi_{-} I \otimes \sigma_{+}+(\Delta / 2) \sigma_{z} \otimes I+$ $\gamma_{1} / 2\left[\sigma_{x} \otimes \sigma_{x}+\sigma_{y} \otimes \sigma_{y}\right]$, where $\sigma_{i}$ and $I$ are the Pauli and identity matrices, respectively. We defined $\sigma_{ \pm} \equiv$ $\frac{1}{2}\left(\sigma_{x} \pm i \sigma_{y}\right)$ and $\pi_{ \pm} \equiv \hbar\left(k_{x} \pm i k_{y}\right)$. Here, $v_{f}$ is the in-plane velocity [40] and $\Delta$ is the electrostatic potential difference between the two layers. Expressions for noninteracting ground-state electronic bands $\xi_{n}(\mathbf{k})(n=1-4$, see inset of Fig. 1) and wave functions $\Phi_{n}(\mathbf{k})$ are obtained by diagonalizing $\mathcal{H}_{k}$; see the Supplemental Material [41]

We consider coupling of long wavelength longitudinal or transverse optical (LO/TO) phonons near the $\Gamma$ point with the graphene plasmons. Relative displacement of the two sublattice in the top layer $(T)$ is given by

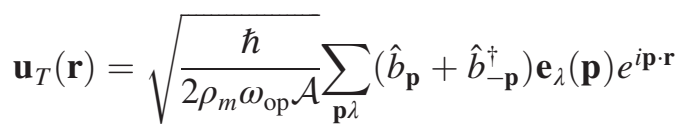

where $\mathcal{A}$ is the area of the unit cell, $\rho_{m}$ is the mass density of graphene, $\mathbf{p}=\left(p_{x}, p_{y}\right)$ is the phonon wave vector, $\lambda$ denotes the LO/TO modes where $\hat{b}_{\mathbf{p} \lambda}^{\dagger}$ are its creation operators, $\mathbf{e}_{\lambda}(\mathbf{p})$ are the polarization vectors given by $\mathbf{e}_{\mathrm{LO}}(\mathbf{p})=i(\cos \varphi, \sin \varphi)$, and $\mathbf{e}_{\mathrm{TO}}(\mathbf{p})=i(-\sin \varphi, \cos \varphi)$ where $\varphi=\tan ^{-1}\left(p_{y} / p_{x}\right)$. Because of the two graphene 


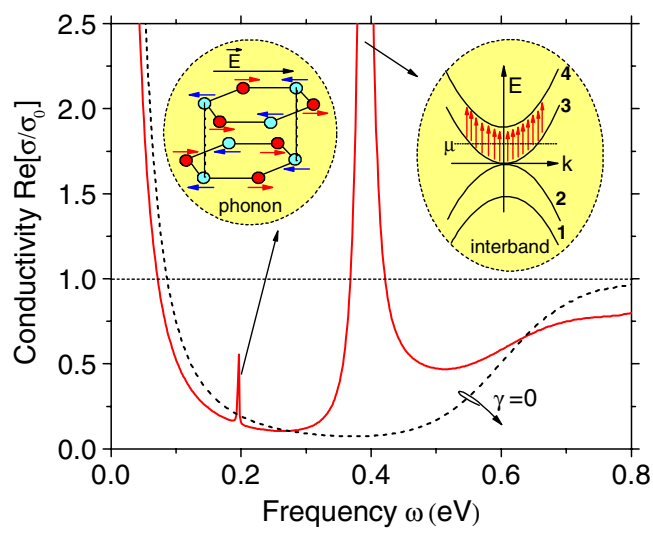

FIG. 1 (color online). Real part of bulk bilayer graphene conductivity (solid line) computed at $T=300 \mathrm{~K}$ at chemical potential of $\mu=0.3 \mathrm{eV}$, constant damping of $\eta=10 \mathrm{meV}$, zero gap (i.e., $\Delta=0 \mathrm{eV}$ ), and $q=0$. This is compared with the case where $\gamma_{1}=0 \mathrm{eV}$ (dashed line); $\sigma_{0}$ is the universal conductivity of $e^{2} / 2 \hbar$.

layers, there are two possible vibrational modes, i.e., symmetric $\left[\mathbf{u}_{B}(\mathbf{r})=\mathbf{u}_{T}(\mathbf{r})\right]$ and antisymmetric $\left[\mathbf{u}_{B}(\mathbf{r})=\right.$ $-\mathbf{u}_{T}(\mathbf{r})$ ], where subscript $B$ denotes the bottom layer. Hence, the electron-phonon coupling at the $\mathbf{K}$ valley for bilayer graphene is given by $[42,43]$

$$
H_{e-\mathrm{op}}(\mathbf{r})=-\sqrt{2} \frac{2 \beta \hbar v_{F}}{3 a^{2}} \boldsymbol{\sigma}^{ \pm} \times \mathbf{u}(\mathbf{r})
$$

with $a \approx 1.4 \AA$ as the $\mathrm{C}-\mathrm{C}$ distance, $\sigma_{j}^{+}=I \sigma_{j}, \sigma_{j}^{-}=\sigma_{z} \sigma_{j}$, and where $\beta=-\partial \ln \gamma_{0} / \partial a$ is a dimensionless parameter related to the deformation potential. Without loss of generality, we take the electric field polarization to be along $y$ and $\varphi=0$. Since only the lattice vibration along $y$ can couple to light, we consider only the TO lattice mode. As a result, we can write the electron-phonon interaction for the $v$ mode in the following form

$$
\mathcal{H}_{v}^{\prime}=\frac{1}{\sqrt{\mathcal{A}}} \sum_{\mathbf{k}} \hat{a}_{\mathbf{k}+\mathbf{p}}^{\dagger} \mathcal{V}_{v}(\mathbf{p}) \hat{a}_{\mathbf{k}} e^{i \mathbf{p} \cdot \mathbf{r}}\left(\hat{b}_{\mathbf{p}}+\hat{b}_{\mathbf{p}}^{\dagger}\right)
$$

where $v=S, A$ denotes the symmetric and antisymmetric modes, with $\mathcal{V}_{S}(\mathbf{p} \rightarrow 0)=i g I \sigma_{x} \quad$ and $\quad \mathcal{V}_{A}(\mathbf{p} \rightarrow 0)=$ $i g \sigma_{z} \sigma_{x}$, where

$$
g \equiv \frac{\beta \hbar v_{F}}{L^{2}} \sqrt{\frac{\hbar}{2 \rho_{m} \omega_{\mathrm{op}}}} \approx 0.3 \mathrm{eV} \AA^{-1},
$$

since $\beta \approx 2$ and $\hbar \omega_{\mathrm{op}} \approx 0.2 \mathrm{eV}$ [42].

The plasmonic response of bilayer graphene can be obtained from its dielectric function given by

$$
\epsilon_{T}^{\mathrm{rpa}}(q, \omega)=\kappa-v_{c} \Pi_{\rho, \rho}^{0}(q, \omega)-v_{c} \frac{q^{2}}{\omega^{2}} \delta \Pi_{j, j}(q, \omega),
$$

at arbitrary wave vector $q$ and frequency $\omega ; v_{c}=e^{2} / 2 q \epsilon_{0}$ is the two-dimensional (2D) Coulomb interaction, and $\kappa$ is the effective dielectric constant of the environment. Here,
$\Pi_{\rho, \rho}^{0}(q, \omega)$ is the noninteracting part (i.e., the pair bubble diagram) of the charge-charge correlation function given by $[11,12]$

$$
\begin{aligned}
\Pi_{\rho, \rho}^{0}(q, \omega)= & -\frac{g_{s} g_{v}}{(2 \pi)^{2}} \sum_{n n^{\prime}} \int d \mathbf{k} \\
& \times \frac{n_{F}\left(\xi_{n}(\mathbf{k})\right)-n_{F}\left(\xi_{n^{\prime}}(\mathbf{k}+\mathbf{q})\right)}{\xi_{n}(\mathbf{k})-\xi_{n^{\prime}}(\mathbf{k}+\mathbf{q})+\hbar \omega+i \hbar / \tau_{e}}\left|F_{n n^{\prime}}(\mathbf{k}, \mathbf{q})\right|^{2},
\end{aligned}
$$

where $n_{F}$ is the Fermi-Dirac distribution function, $g_{s}$ and $g_{v}$ are the spin or valley degeneracies, $F_{n n^{\prime}}(\mathbf{k}, \mathbf{q})=$ $\left\langle\Phi_{n}(\mathbf{k}) \mid \Phi_{n^{\prime}}(\mathbf{k}+\mathbf{q})\right\rangle$ is the band overlap, and $\tau_{e}$ is the electron lifetime, where we assumed a typical experimental value of $\eta \equiv \hbar / \tau_{e} \approx 10 \mathrm{meV}$ [19].

The effect of electron-phonon interaction is included within $\delta \Pi_{j, j}(q, \omega)$, where subscript $j$ denotes the current operator. Here, we employ a model for $\delta \Pi_{j, j}(q, \omega)$ that is consistent with the various electron-phonon selection rules for the symmetric or antisymmetric modes and Fano effect observed in optical spectroscopy experiments for bilayer graphene. The detailed implementation follows a formalism known as the charged-phonon theory [44-46],

$$
\delta \Pi_{j, j}(q, \omega)=\sum_{v v^{\prime}} \Gamma_{j, v}(q, \omega) \mathcal{D}_{v v^{\prime}}(\omega) \Gamma_{v^{\prime \dagger}, j}(q, \omega),
$$

where

$$
\begin{aligned}
\Gamma_{j, v}(q, \omega)= & -\frac{g_{s} g_{v}}{(2 \pi)^{2}} \sum_{n n^{\prime}} \int d \mathbf{k} \\
& \times \frac{n_{F}\left(\xi_{n}(\mathbf{k})\right)-n_{F}\left(\xi_{n}(\mathbf{k}+\mathbf{q})\right)}{\xi_{n}(\mathbf{k})-\xi_{n^{\prime}}(\mathbf{k}+\mathbf{q})+\hbar \omega+i \hbar / \tau_{e}}[\mathcal{J}]_{n n^{\prime}}\left[\mathcal{V}_{v}\right]_{n^{\prime} n},
\end{aligned}
$$

where $\quad[\mathcal{J}]_{n n^{\prime}}=\left\langle\Phi_{n}(\mathbf{k})|\mathcal{J}| \Phi_{n^{\prime}}(\mathbf{k}+\mathbf{q})\right\rangle \quad$ and $\quad\left[\mathcal{V}_{v}\right]_{n n^{\prime}}=$ $\left\langle\Phi_{n}(\mathbf{k})\left|\mathcal{V}_{v}\right| \Phi_{n^{\prime}}(\mathbf{k}+\mathbf{q})\right\rangle$ with $v=A, S$ and the current operator defined as $\mathcal{J} \equiv v_{F} I \sigma_{y}$ with the direction of the electric field. $\mathcal{D}$ is the phonon Green's function,

$$
\left[\mathcal{D}^{-1}(\omega)\right]_{v v^{\prime}}=\delta_{v v^{\prime}}\left[\mathcal{D}_{0}^{-1}(\omega)\right]-\Gamma_{v^{\dagger}, v^{\prime}}(\omega)
$$

where $\mathcal{D}_{0}=2 \omega_{\mathrm{op}} / \hbar\left(\left(\omega+i / \tau_{\mathrm{op}}\right)^{2}-\omega_{\mathrm{op}}^{2}\right)$ is the free phonon Green's function and $\tau_{\mathrm{op}}$ describes the phonon lifetime. In this calculation, we assumed $\tau_{\text {op }} \approx 1$ ps [47].

Figure 1 shows the optical conductivity of bilayer graphene calculated from the relation [45]

$$
\sigma(q, \omega)=\underbrace{i \frac{e^{2} \omega}{q^{2}} \Pi_{\rho, \rho}^{0}(q, \omega)}_{\bar{\sigma}}+\underbrace{i \frac{e^{2}}{\omega} \delta \Pi_{j, j}(q, \omega)}_{\delta \sigma} .
$$

The calculation assumes $T=300 \mathrm{~K}$, chemical potential of $\mu=0.3 \mathrm{eV}$, and $\Delta=0 \mathrm{eV} ; \bar{\sigma}$ is the noninteracting optical conductivity, which accounts for a Drude peak at $\omega=0$ 
and a universal conductivity of $e^{2} / 2 \hbar$. The peak conductivity at $\hbar \omega=\gamma$ is due to interband transitions between two perfectly nested bands, e.g., $\xi_{3}$ and $\xi_{4}$, separated in energy by $\gamma$; see inset. These conductivity peaks at $\omega=0$ and $\hbar \omega=\gamma$ are phenomenologically broadened by $\omega \rightarrow \omega+i / \tau_{e}$ in the model; $\delta \sigma$ accounts for the electronic interaction with the IR phonons modes $(v=A, S)$ and agrees well with experimentally measured optical spectra of bilayer graphene [45]. In our zero gap case, only the $A$ mode (asymmetric mode) is IR active [45]; see the inset of Fig. 1. This mode is responsible for the sharp resonance feature at $\omega=\omega_{\mathrm{op}}$.

Longitudinal collective plasmonic dispersion is obtained by looking for the zeros in the real part of the dynamical dielectric function, i.e., $\operatorname{Re}\left[\epsilon_{T}^{\mathrm{rpa}}(q, \omega)\right]=0$. For bilayer graphene, there are three solutions [24,48], a "classical" plasmon with $\sqrt{q}$ behavior, an acoustic plasmon with $\propto q$ behavior, and a high-energy $\gamma$ plasmon residing near the interband resonance $\gamma$. Only the former has been found to be fully coherent, whose dispersion in the long wavelength limit can be shown to follow

$$
\omega_{p l}(q)=\frac{1}{\hbar} \sqrt{\frac{q e^{2} g}{4 \pi \epsilon_{0} \kappa} \sum_{j} \frac{n_{j}(\mu)}{D_{j}(\mu)}}
$$

where $g=4$ is the degeneracy factor and $n_{j}(\mu)$ and $D_{j}(\mu)$ are the carrier density and density of states of the $j$ th band, respectively. On the other hand, the other two solutions are overdamped. The acoustic plasmon lies in the intraband continuum and is always overdamped with insignificant spectral weight $[24,48]$. Under typical conditions, the highenergy $\gamma$ plasmon is also overdamped, lying in the interband continuum (i.e., $\xi_{1}, \xi_{2} \rightarrow \xi_{3}, \xi_{4}$ transitions) when $2 \mu<\gamma$ and the low-energy interband continuum (i.e., $\xi_{1} \rightarrow \xi_{2}$ or $\xi_{3} \rightarrow \xi_{4}$ transitions) when $2 \mu>\gamma$. We show later that, under certain conditions, this mode can become fully coherent.

Electron loss function, defined as the imaginary part of the inverse dielectric function, i.e., $L(q, \omega)=$ $\left[\epsilon_{T}^{\mathrm{rpa}}(q, \omega)\right]^{-1}$, is a quantity that can be probed in various spectroscopy experiments $[19,49,50]$. Figure 2(a) shows the calculated $L(q, \omega)$ assuming typical experimental conditions: $\mu=0.3 \mathrm{eV}, \Delta=0 \mathrm{eV}, T=300 \mathrm{~K}, \kappa=2.5$, and $\eta=10 \mathrm{meV}$. The single particle continuums are also indicated: (1) intraband, (2) electron-hole interband, and (3) low-energy interband. The $\sqrt{q}$ plasmon lies above the intraband continuum and compares well with the long wavelength dispersion $\omega_{p l}(q)$, while the $\gamma$ plasmon is significantly broadened. The most important result is the appearance of distinctively sharp structure near $\omega \approx \omega_{\text {op }}$, not seen in monolayer graphene [11,12].

Figure 2(b) plots the loss spectra at different momenta $q$. We observe an enhancement in the IR activity of the phonon mode as the plasmon resonance approaches $\omega_{\mathrm{op}}$. The transfer of plasmonic spectral weight to the IR phonon mode, as reflected by an increase in both intensity and linewidth, is enhanced with decreasing detuning. Renormalized by many-body interactions, this "dressed"
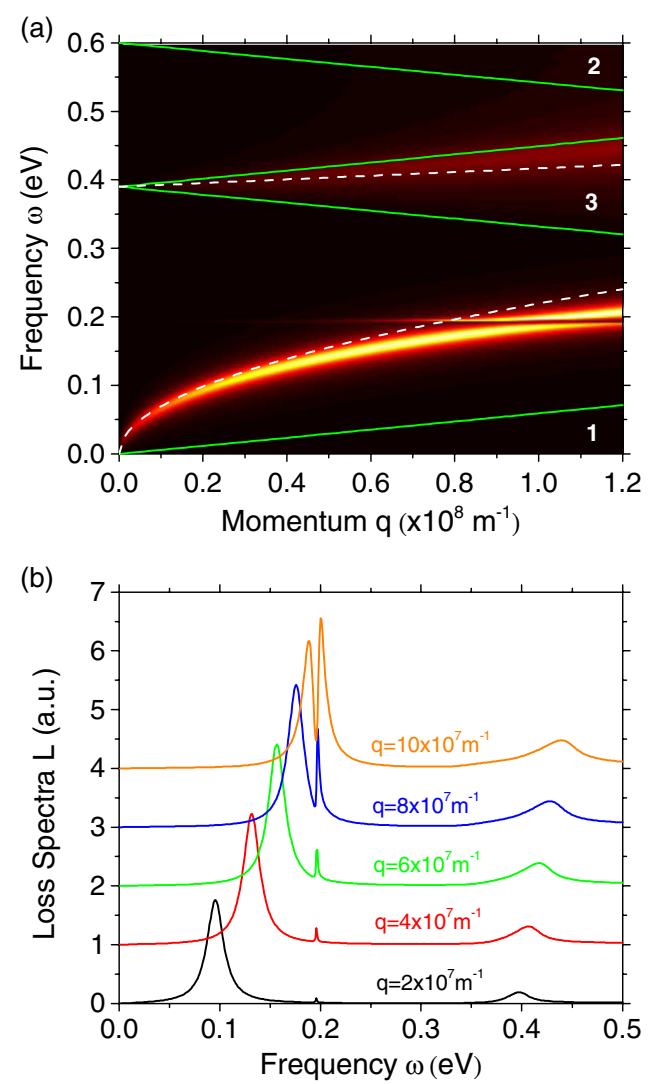

FIG. 2 (color online). (a) RPA electron loss function $L(q, \omega)$ for bilayer graphene computed at $T=300 \mathrm{~K}$ at chemical potential of $\mu=0.3 \mathrm{eV}$, constant damping of $\eta=10 \mathrm{meV}$, zero energy gap (i.e., $\Delta=0 \mathrm{eV}$ ), and a background dielectric constant of $\kappa=2.5$. Green lines are boundaries for the Landau damped regions. (b) Plots of spectra at different plasmon momenta $q$.

phonon exhibits pronounced IR activity and is also accompanied by Fano asymmetric spectral line shapes. The Fano feature is acquired through interference between the discrete phonon mode and the "leaky" plasmonic mode; the electronic lifetime is significantly shorter than that of the phonon, broadening the former into a quasicontinuum. The loss spectra show the evolution of the plasmonic and phonon resonances as they approach each other. They evolve from separate resonances at small $q$ to a Fano line shape and eventually an induced narrow transparency at zero detuning. This very narrow transparent window emerged within the broadly opaque plasmonic absorption, a phenomenon analogous to the electromagnetically induced transparency [5], and should also be accompanied by novel electromagnetic effects such as slow light [51]. On the contrary, plasmon coupling with substrate surface optical phonons typically leads to well-separated resonances instead $[19,29]$.

Transmission spectroscopy studies have proven to be very effective in probing the plasmonic properties of graphene, where finite plasmon momentum $q$ can be sampled by simply patterning graphene into nanostructures $[17,18]$. Graphene nanostructures with dimensions down to $100 \mathrm{~nm}$ would allow us to access these predicted mid-IR plasmonic features under 
experimentally accessible doping conditions [18]. The enhancement of IR phonon activity with decreased detuning between the phonon and plasmon resonance might lead to interesting applications. Indeed, such plasmon-enhanced IR absorption has permitted an emerging field of spectroscopy by noble metals of surfaces and electrochemical systems [52]. Tunable plasmonic resonance in graphene nanostructured surfaces might allow for the detection of molecules through enhancement of its IR vibrational modes.

Previously, we have seen that the $\gamma$-plasmon mode is overdamped. In the limit of small momenta, it has the following dispersion [48]

$$
\omega_{\gamma}(q)=\frac{1}{\hbar}\left[\gamma+\frac{q e^{2}}{8 \pi \epsilon_{0} \kappa} \log \left(1+2 \frac{\mu}{\gamma}\right)\right] .
$$

If the $\gamma$ plasmon gains sufficient oscillator strength, e.g., by modifying its doping $(\uparrow \mu)$ or dielectric environment $(\downarrow \kappa)$, it can reside outside the low-energy interband continuum. This is shown in Fig. 3(a) (dashed line), calculated using Eq. (12) assuming $\mu=0.6 \mathrm{eV}$ and $\kappa=1$. The electron loss function in Fig. 3(a) indicates several interesting features of this high-energy $\gamma$-plasmon mode. First, its dispersion departs from the simple $\omega_{\gamma}-\gamma \propto q$ relation, acquiring an increasingly $q^{2}$ behavior with $q$. We find that the modified dispersion can be described within a model that accounts for the effective coupling between the classical and $\gamma$ plasmon as follows:

$$
\epsilon_{\mathrm{eff}} \approx \kappa\left[1-\frac{\omega_{p l}^{2}}{\omega^{2}}-\frac{\alpha^{2}}{\omega^{2}-\omega_{\gamma}^{2}+\alpha^{2}}\right]
$$

where $\alpha$ is an effective coupling between the two modes. Using the long-wavelength expressions for these modes, i.e., Eqs. (11) and (12) (dashed white lines) and a coupling energy $\alpha=85 \mathrm{meV}$, the coupled mode solutions (solid white lines) obtained by solving for $\epsilon_{\text {eff }}=0$ agree well with the dispersions observed in the loss function. Second, we observed prominent spectral weight transfer from the conventional 2D plasmon to the $\gamma$-plasmon mode.

Figure 3(b) plots the calculated $L(q, \omega)$ and $L(q, \omega) / \omega$ spectra at typical values of $q=2-10 \times 10^{7} \mathrm{~m}^{-1}$. The integrated loss function $\int_{0}^{\infty} L(q, \omega) d \omega$ is related to the Coulomb energy stored in the electron fluid [53]. On the other hand, through the Kramers-Kronig relations, one can obtain the sum rule $\int_{0}^{\infty} L(q, \omega) / \omega d \omega=-1 / \pi$ [54], with conserved spectral weight at different $q$. We see that the $\gamma$ plasmon acquires a spectral weight an order of magnitude larger than the conventional plasmon as the latter enters into the Landau damped region. Hence, it should be experimentally observable. The possibility of an "optical"-like high-energy plasmonic mode, previously presumed to be overdamped with little spectral weight [48], might open up applications in the higher mid-IR spectral range. With high enough doping, e.g., with electrolyte gating, this mode can gain enough oscillator
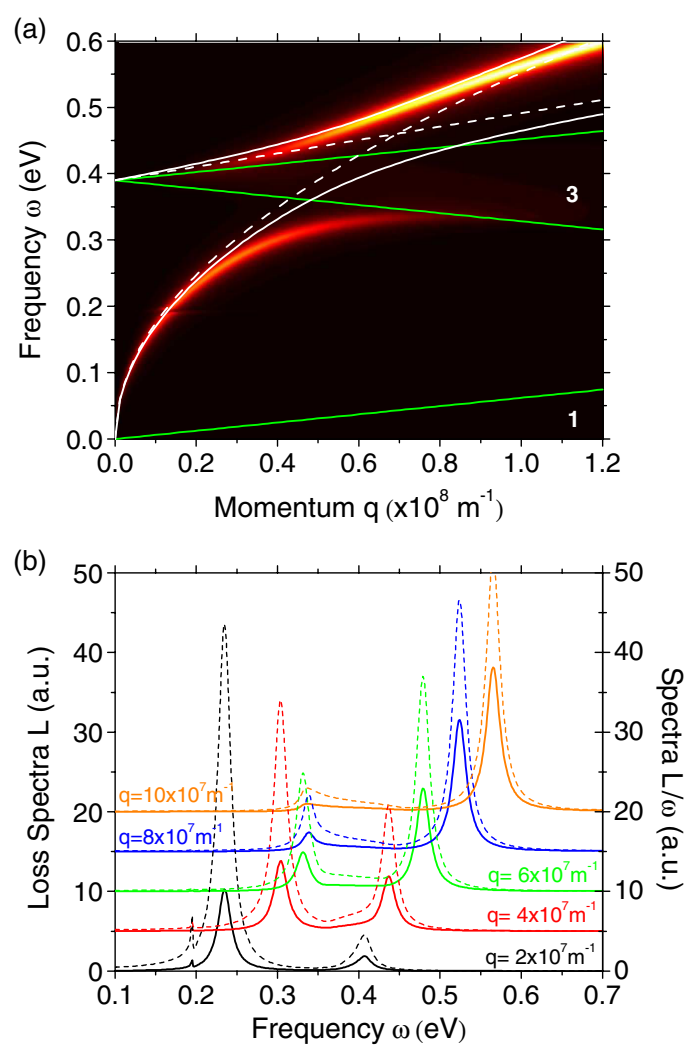

FIG. 3 (color online). (a) RPA electron loss function $L(q, \omega)$ for bilayer graphene computed at $T=300 \mathrm{~K}$ at chemical potential of $\mu=0.6 \mathrm{eV}$, constant damping of $\eta=10 \mathrm{meV}$, zero energy gap (i.e., $\Delta=0 \mathrm{eV}$ ), and a background dielectric constant of $\kappa=1$. (b) Plots of spectra $L$ (solid lines) and $L / \omega$ (dashed lines) at different plasmon momenta $q$.

strength and be pushed out of the Landau damped region, to become a coherent plasmonic mode.

In summary, we have shown that bilayer graphene as a new plasmonic material is important and interesting in its own right. The above-mentioned new mid-IR plasmonic effects can also be generalized to more complex graphene stacks [55], for example, $A B C$ or $A B A$ trilayers. These new plasmonic resonant features can also potentially lead to interesting applications such as engineered metamaterials with novel electromagnetic effects [56], resonant heat transfer processes [57], and many others [5].

F. G. acknowledges financial support from the Spanish Ministry of Economy (MINECO) through Grant No. FIS2011-23713, from the European Research Council Advanced Grant, Contract No. 290846, and from the European Commission under the Graphene Flagship Contract No. CNECT-ICT-604391.

*tonyaslow@gmail.com

[1] S. A. Maier, Plasmonics: Fundamentals and Applications (Springer, New York, 2007). 
[2] D. Pines, Elementary Excitations in Solids: Lectures on Phonons, Electrons, and Plasmons, Vol. 5 (Westview Press, Boulder, 1999).

[3] D. K. Gramotnev and S. I. Bozhevolnyi, Nat. Photonics 4, 83 (2010).

[4] V. M. Shalaev, Nat. Photonics 1, 41 (2007).

[5] B. Luk'yanchuk, N. I. Zheludev, S. A. Maier, N. J. Halas, P. Nordlander, H. Giessen, and C. T. Chong, Nat. Mater. 9, 707 (2010).

[6] A. Kabashin, P. Evans, S. Pastkovsky, W. Hendren, G. Wurtz, R. Atkinson, R. Pollard, V. Podolskiy, and A. Zayats, Nat. Mater. 8, 867 (2009).

[7] A. Grigorenko, M. Polini, and K. Novoselov, Nat. Photonics 6, 749 (2012).

[8] T. Low and P. Avouris, ACS Nano 8, 1086 (2014).

[9] M. Jablan, M. Soljacic, and H. Buljan, Phys. Rev. B 83, 161409(R) (2011).

[10] F. Koppens, D. E. Chang, and F. J. G. de Abajo, Nano Lett. 11, 3370 (2011).

[11] E. H. Hwang and S. Das Sarma, Phys. Rev. B 75, 205418 (2007).

[12] B. Wunsch, T. Stauber, F. Sols, and F. Guinea, New J. Phys. 8, 318 (2006).

[13] A. Y. Nikitin, F. Guinea, F. J. Garcia-Vidal, and L. MartinMoreno, Phys. Rev. B 85, 081405(R) (2012).

[14] A. Y. Nikitin, F. Guinea, F. J. García-Vidal, and L. MartínMoreno, Phys. Rev. B 84, 161407 (2011).

[15] Z. Fei, A. S. Rodin, G. O. Andreev, W. Bao, A. S. McLeod, M. Wagner, L. M. Zhang, Z. Zhao, M. Thiemens, G. Dominguez et al., Nature (London) 487, 82 (2012).

[16] J. Chen, M. Badioli, P. Alonso-Gonzalez, S. Thongrattanasiri, F. Huth, J. Osmond, M. Spasenovic, A. Centeno, A. Pesquera, P. Godignon et al., Nature (London) 487, 77 (2012).

[17] L. Ju, B. Geng, J. Horng, C. Girit, M. Martin, Z. Hao, H. A. Bechtel, X. Liang, A. Zettl, Y. R. Shen et al., Nat. Nanotechnol. 6, 630 (2011).

[18] H. Yan, X. Li, B. Chandra, G. Tulevski, Y. Wu, M. Freitag, W. Zhu, P. Avouris, and F. Xia, Nat. Nanotechnol. 7, 330 (2012).

[19] H. Yan, T. Low, W. Zhu, Y. Wu, M. Freitag, X. Li, F. Guinea, P. Avouris, and F. Xia, Nat. Photonics 7, 394 (2013).

[20] E. Carrasco, M. Tamagnone, and J. Perruisseau-Carrier, Appl. Phys. Lett. 102, 104103 (2013).

[21] L. Wu, H. Chu, W. Koh, and E. Li, Opt. Express 18, 14395 (2010).

[22] M. Freitag, T. Low, W. Zhu, H. Yan, F. Xia, and P. Avouris, Nat. Commun. 4, 1951 (2013).

[23] M. Freitag, T. Low, F. Xia, and P. Avouris, Nat. Photonics 7 53 (2012).

[24] R. Sensarma, E. H. Hwang, and S. Das Sarma, Phys. Rev. B 82, 195428 (2010).

[25] O. V. Gamayun, Phys. Rev. B 84, 085112 (2011).

[26] E. V. Gorbar, V. P. Gusynin, and V. A. Miransky, Phys. Rev. B 81, 155451 (2010).

[27] G. Borghi, M. Polini, R. Asgari, and A. H. MacDonald, Phys. Rev. B 80, 241402 (2009).

[28] S. V. Kusminskiy, D. Campbell, and A. C. Neto, Europhys. Lett. 85, 58005 (2009).

[29] E. H. Hwang, R. Sensarma, and S. Das Sarma, Phys. Rev. B 82, 195406 (2010).
[30] T.-T. Tang, Y. Zhang, C.-H. Park, B. Geng, C. Girit, Z. Hao, M. C. Martin, A. Zettl, M. F. Crommie, S. G. Louie et al., Nat. Nanotechnol. 5, 32 (2009).

[31] A. B. Kuzmenko, L. Benfatto, E. Cappelluti, I. Crassee, D. van der Marel, P. Blake, K. S. Novoselov, and A. K. Geim, Phys. Rev. Lett. 103, 116804 (2009).

[32] J. Nilsson, A.H. Castro Neto, F. Guinea, and N. M. R. Peres, Phys. Rev. Lett. 97, 266801 (2006).

[33] D. S. L. Abergel and V. I. Falko, Phys. Rev. B 75, 155430 (2007).

[34] E. J. Nicol and J. P. Carbotte, Phys. Rev. B 77, 155409 (2008).

[35] F. Wang, Y. Zhang, C. Tian, C. Girit, A. Zettl, M. Crommie, and Y. R. Shen, Science 320, 206 (2008).

[36] A. B. Kuzmenko, E. van Heumen, D. van der Marel, P. Lerch, P. Blake, K. Novoselov, and A. K. Geim, Phys. Rev. B 79, 115441 (2009).

[37] Z. Q. Li, E. A. Henriksen, Z. Jiang, Z. Hao, M. C. Martin, P. Kim, H. L. Stormer, and D. N. Basov, Phys. Rev. Lett. 102, 037403 (2009).

[38] T. Ohta, A. Bostwick, T. Seyller, K. Horn, and E. Rotenberg, Science 313, 951 (2006).

[39] S. Zhou, D. Siegel, A. Fedorov, F. El Gabaly, A. Schmid, A. C. Neto, D.-H. Lee, and A. Lanzara, Nat. Mater. 7, 259 (2008).

[40] E. McCann, Phys. Rev. B 74, 161403(R) (2006).

[41] See Supplemental Material at http://link.aps.org/ supplemental/10.1103/PhysRevLett.112.116801 for expressions of the eigen-energies and wavefunctions of bilayer graphene.

[42] T. Ando, J. Phys. Soc. Jpn. 76, 104711 (2007).

[43] A. H. Castro Neto and F. Guinea, Phys. Rev. B 75, 045404 (2007).

[44] M. J. Rice and H.-Y. Choi, Phys. Rev. B 45, 10173 (1992).

[45] E. Cappelluti, L. Benfatto, and A. B. Kuzmenko, Phys. Rev. B 82, 041402(R) (2010).

[46] E. Cappelluti, L. Benfatto, M. Manzardo, and A. B. Kuzmenko, Phys. Rev. B 86, 115439 (2012).

[47] N. Bonini, M. Lazzeri, N. Marzari, and F. Mauri, Phys. Rev. Lett. 99, 176802 (2007).

[48] O. V. Gamayun, Phys. Rev. B 84, 085112 (2011).

[49] T. Eberlein, U. Bangert, R. R. Nair, R. Jones, M. Gass, A. L. Bleloch, K. S. Novoselov, A. Geim, and P. R. Briddon, Phys. Rev. B 77, 233406 (2008).

[50] G. Abstreiter, M. Cardona, and A. Pinczuk, Light Scattering by Free Carrier Excitations in Semiconductors (Springer, New York, 1984).

[51] M. Sandtke and L. Kuipers, Nat. Photonics 1, 573 (2007).

[52] R. F. Aroca, D. J. Ross, C. Domingo et al., Appl. Spectrosc. 58, 324A (2004).

[53] D. Pines and P. Nozieres, The Theory of Quantum Fluids (Westview Press, Boulder, 1966).

[54] D. van der Marel, Optical Signatures of Electron Correlations in the Cuprates (Kluwer Academic Publishers, Dordrecht, 2005).

[55] F. Guinea, A. H. Castro Neto, and N. M. R. Peres, Phys. Rev. B 73, 245426 (2006).

[56] H. Yan, T. Low, F. Guinea, F. Xia, and P. Avouris, arXiv:1310.4394.

[57] S. Shen, A. Narayanaswamy, and G. Chen, Nano Lett. 9, 2909 (2009). 